\title{
Spatial Relationship Between Coronary Microvascular Dysfunction and Delayed Contrast Enhancement in Patients with Hypertrophic Cardiomyopathy
}

\author{
Barbara Sotgia ${ }^{1}$, Roberto Sciagrà ${ }^{1}$, Iacopo Olivotto ${ }^{2}$, Giancarlo Casolo ${ }^{3}$, Luigi Rega ${ }^{4}$, Irene Betti ${ }^{4}$, Alberto Pupi ${ }^{1}$, \\ Paolo G. Camici ${ }^{5}$, and Franco Cecchi ${ }^{2}$ \\ ${ }^{1}$ Department of Clinical Physiopathology_Nuclear Medicine Unit, Azienda Ospedaliera Universitaria Careggi, Florence, Italy; \\ ${ }^{2}$ Regional Referral Center for Myocardial Diseases, Azienda Ospedaliera Universitaria Careggi, Florence, Italy; ${ }^{3}$ Division of \\ Cardiology, Ospedale Versilia, Lido di Camaiore, Italy ; ${ }^{4}$ Centre for Magnetic Resonance, Azienda Ospedaliera Universitaria \\ Careggi, Florence, Italy; and ${ }^{5}$ Medical Research Council Clinical Sciences Centre, Hammersmith Hospital, Imperial College, \\ London, United Kingdom
}

To clarify the spatial relationship between coronary microvascular dysfunction and myocardial fibrosis in hypertrophic cardiomyopathy $(\mathrm{HCM})$, we compared the measurement of hyperemic myocardial blood flow (hMBF) by PET with the extent of delayed contrast enhancement (DCE) detected by MRI. Methods: In 34 patients with HCM, PET was performed using ${ }^{13} \mathrm{~N}$-labeled ammonia during hyperemia induced by intravenous dipyridamole. DCE and systolic thickening were assessed by MRI. Left ventricular myocardial segments were classified as with DCE, either transmural (DCE-T) or nontransmural (DCE-NT), and without DCE, either contiguous to DCE segments (NoDCE-C) or remote from them (NoDCE-R). Results: In the group with DCE, hMBF was significantly lower than in the group without DCE $(1.81 \pm$ 0.94 vs. $2.13 \pm 1.11 \mathrm{~mL} / \mathrm{min} / \mathrm{g} ; P<0.001)$. DCE-T segments had lower hMBF than did DCE-NT segments $(1.43 \pm 0.52$ vs. $1.91 \pm 1 \mathrm{~mL} / \mathrm{min} / \mathrm{g}, P<0.001)$. Similarly, NoDCE-C segments had lower hMBF than did NoDCE-R $(1.98 \pm 1.10$ vs. $2.29 \pm$ $1.10 \mathrm{~mL} / \mathrm{min} / \mathrm{g}, P<0.01$ ) and had no significant difference from DCE-NT segments. Severe coronary microvascular dysfunction (hMBF in the lowest tertile of all segments) was more prevalent among NoDCE-C than NoDCE-R segments (33\% vs. $24 \%, P<$ $0.05)$. Systolic thickening was inversely correlated with percentage transmurality of DCE (Spearman $\rho=-0.37, P<0.0001$ ) and directly correlated with hMBF (Spearman $\rho=0.20, P<$ 0.0001). Conclusion: In myocardial segments exhibiting DCE, $\mathrm{hMBF}$ is reduced. DCE extent is inversely correlated and hMBF directly correlated with systolic thickening. In segments without DCE but contiguous to DCE areas, hMBF is significantly lower than in those remote from DCE and is similar to the value obtained in nontransmural DCE segments. These results suggest that increasing degrees of coronary microvascular dysfunction might play a causative role for myocardial fibrosis in $\mathrm{HCM}$.

Received Dec. 20, 2007; revision accepted Mar. 6, 2008.

For correspondence or reprints contact: Roberto Sciagrà, Nuclear Medicine, Department of Clinical Physiopathology, University of Florence; Viale Morgagni 85; 50134 Florence, Italy.

E-mail: r.sciagra@dfc.unifi.it

COPYRIGHT @ 2008 by the Society of Nuclear Medicine, Inc.
Key Words: coronary microvascular dysfunction; fibrosis; hyperthrophic cardiomyopathy; myocardial blood flow; positron emission tomography

J Nucl Med 2008; 49:1090-1096

DOI: 10.2967/jnumed.107.050138

$\mathbf{H}$ ypertrophic cardiomyopathy (HCM) is a genetically determined cardiac disease with notable heterogeneity in clinical expression, natural history, and prognosis $(1,2)$. $\mathrm{HCM}$ is defined anatomically by typical histologic features: small-vessel disease, characterized by medial hypertrophy and narrowing of the intramural coronary vessels (3-5); myocite disarray; and fibrosis $(6,7)$. Some of these histologic substrates are responsible for the distinctive microvascular dysfunction and myocardial scarring that are commonly encountered features in patients with HCM. Coronary microvascular dysfunction represents a predisposing factor for myocardial ischemia (8-12) and is a strong, independent predictor of long-term clinical progression, left ventricular (LV) remodeling, and cardiovascular death as demonstrated by the adverse prognostic meaning of a blunted hyperemic myocardial blood flow (hMBF) $(13,14)$. Similarly, the extent of myocardial scarring is related to LV functional impairment and may identify patients at risk of disease progression and increased arrhythmic risk $(15,16)$.

The gold standard for the evaluation of coronary microvascular dysfunction is represented by PET, which allows the quantitative assessment of MBF per unit of myocardial mass (12,17-21). Myocardial scarring can be visualized as delayed contrast enhancement (DCE) within the left ventricle by MRI $(15,16,22)$. To date, few studies have compared baseline MBF and DCE in HCM $(23,24)$, and to our knowledge, no data assessing the spatial relationship between 
hMBF and DCE exist. Therefore, the pathophysiologic relationship between coronary microvascular dysfunction and fibrosis remains elusive. We hypothesized that, at least in part, myocardial scarring in HCM could be secondary to ischemia due to coronary microvascular dysfunction. To evaluate this hypothesis, we examined the relationship of DCE to hMBF abnormalities in an HCM cohort from a tertiary referral center.

\section{MATERIALS AND METHODS}

\section{Patients with HCM}

The study cohort included 34 patients $(23$ men and 11 women; mean age $\pm \mathrm{SD}, 37 \pm 17 \mathrm{y}$, range, $15-71 \mathrm{y}$ ) from a large HCM population, closely followed by physicians with long-standing interest in HCM at our institution. The HCM diagnosis was made on the basis of 2-dimensional echocardiographic evidence of a hypertrophied left ventricle (maximal wall thickness $\geq 15 \mathrm{~mm}$ ) in the absence of any other cardiac or systemic cause of LV hypertrophy $(1,2)$. For all patients, informed consent to participate in the study was obtained, and the study protocol was approved by the local ethics committee. All patients underwent baseline echocardiography within 2 wk of the PET scans. Echocardiographic studies were performed with commercially available instruments, using standard views (2). Obstruction of the LV outflow was considered present when a peak outflow gradient of greater than or equal to $30 \mathrm{~mm} \mathrm{Hg}$ was present under basal conditions (25). The characteristics of the patient population are summarized in Table 1.

\section{PET}

All PET scans were performed in the nuclear medicine laboratory (Nuclear Medicine Unit, Department of Clinical Physiopathology, Careggi University Hospital) in Florence between September 2003 and December 2006, after an appropriate period of pharmacologic washout for patients receiving pharmacologic treatment (18). Patients were positioned on the couch of a PET tomographic device (Advance PET; GE Healthcare); a 5-min

TABLE 1

Characteristics of Patient Population

\begin{tabular}{lc}
\hline \multicolumn{1}{c}{ Characteristic } & Data $(n=34)$ \\
\hline Age & $37 \pm 17 \mathrm{y}$ \\
Female & $11(33 \%)$ \\
NYHA functional class (I/II/III) & $17(50 \%) / 16(47 \%) / 1(3 \%)$ \\
Atrial fibrillation & $6(18 \%)$ \\
Echocardiography & \\
$\quad$ LV ventricular wall thickness & $25 \pm 7(15-44)$ \\
$\quad(\mathrm{mm})$ & \\
LV ED diameter $(\mathrm{mm})$ & $23 \pm 5(30-54)$ \\
LV ED diameter $(\mathrm{mm})$ & $3(9 \%)$ \\
LV outflow obstruction & \\
$\quad(\geq 30$ mm Hg) & $122 \pm 29(75-205)$ \\
MRI & $32 \pm 19(10-108)$ \\
LV ED volume $(\mathrm{mL})$ & $74 \pm 12(30-88)$ \\
LV ES volume $(\mathrm{mL})$ & $200 \pm 94(78-547)$ \\
LV ejection fraction $(\%)$ & $21.0 \pm 22.2(0-80)$ \\
LV mass $(\mathrm{g})$ &
\end{tabular}

Data are mean $\pm S D$ (with range in parentheses) or number of patients. transmission scan for attenuation correction was performed using a rod ${ }^{68} \mathrm{Ge}$ source emitting monochromatic $\gamma$-radiation of $511 \mathrm{keV}$ in energy. A complete 2-dimensional set of transmission data was obtained by rotating the ${ }^{68} \mathrm{Ge}$ source around the patient. Additionally, a blank scan was taken to calibrate the transmission scan to correct eventual electronic misalignments in the frame of the postprocessing reconstruction procedure. Then, near-maximal hyperemia was induced by intravenous administration of dipyridamole $(0.56 \mathrm{mg} / \mathrm{kg}$ of body weight over $4 \mathrm{~min})(18,26)$. Three minutes after the end of the dipyridamole infusion, a bolus of $370 \mathrm{MBq}$ of ${ }^{13} \mathrm{~N}$ ammonia ( ${ }^{13} \mathrm{~N}$-labeled ammonia) diluted in $10 \mathrm{~mL}$ of saline solution was intravenously injected over a period of $15-20 \mathrm{~s}$ and followed by a $10-\mathrm{mL}$ saline solution flush at a rate of $2 \mathrm{~mL} / \mathrm{s}$. A dynamic scan with 15 frames of increasing duration $(12 \times 10 \mathrm{~s}, 2 \times 30 \mathrm{~s}$, and $1 \times 60 \mathrm{~s})$ was acquired over $4 \mathrm{~min}$, followed by a prolonged static acquisition of $15 \mathrm{~min}$.

\section{Measurement of hMBF}

Data were analyzed with an operator-interactive computer program (PMOD Cardiac Modeling [PCARD]) (27). Briefly, anatomic images were reconstructed using the static acquisition and reoriented according to the heart axis. After reconstruction, the dynamic images were reoriented as well. On the short-axis slices, regions of interest (ROIs) were manually drawn including the right ventricular cavity, LV cavity, and LV wall from the apex through the base (identified by the appearance of the membranous septum). The ROIs were edited by the standard PMOD volume-ofinterest (VOI) tool to derive the related VOIs. The 3 VOIs were then copied on all ${ }^{13} \mathrm{~N}$-labeled ammonia dynamic images to extract the corresponding time-activity curves. The arterial input function was obtained from the right and LV cavity time-activity curves. The myocardial uptake was derived from the LV wall VOI (27). The LV wall was divided into 17 segments: septal (apical septal, midinferoseptal, midanteroseptal, basal inferoseptal, and basal anteroseptal), anterior (apical anterior, midanterior, and basal anterior), lateral (apical lateral, midlateral, and basal lateral), inferior (apical inferior, midinferolateral, midinferior, basal inferolateral, and basal inferior), and apical (28). Myocardial perfusion was calculated from the dynamic data by fitting the arterial input function and tissue time-activity curves to a 2-tissuecompartment model for ${ }^{13} \mathrm{~N}$ ammonia developed by Hutchins et al. (29). The hMBF for the entire left ventricle was obtained by volume-weighted averaging to the segment territories apex, septum, anterior, lateral, and inferior, as automatically provided by the PCARD program (27). All image studies were analyzed by 1 expert observer, unaware of the patient clinical, echocardiographic, and MRI data.

\section{MRI}

MRI was performed using a 1.5-T scanner (gradient slope, 30 $\mathrm{mT} / \mathrm{m}$ ) (Intera; Philips), with a 5-element phased-array coil. Cine images were acquired in multiple short-axis and long-axis views with a breath-hold retrospective steady-state free precession sequence (slice thickness, $8 \mathrm{~mm}$; echo time, $1.53 \mathrm{~ms}$; matrix, $168 \times$ 195; SENSE factor, 1.8). The number of $\mathrm{k}$-space lines for each heartbeat was adjusted to permit the acquisition of 30 cardiac phases covering the whole cardiac cycle. The field of view was $340 \mathrm{~mm}$ on average and adapted to the size of the patient, leading to a spatial resolution of about $2 \mathrm{~mm}$. A gadolinium-based contrast agent $(0.1 \mathrm{mmol} / \mathrm{kg})$ was then given intravenously, and contrast-enhanced images were acquired by using a 3-dimensional 
breath-hold inversion-recovery segmented gradient-echo sequence in the same views as those used for the cine cardiac magnetic resonance series, 10-15 min after contrast administration (30). The optimal inversion time (IT) was obtained for each patient by visual inspection of a preliminary acquisition of a single-slice multi-IT series. The optimal IT (usually between 175 and $260 \mathrm{~ms}$ ) was the one providing the best contrast between the myocardium and the LV chamber.

\section{MRI Analysis}

Global LV function parameters, including end-diastolic (ED) volume, end-systolic (ES) volume, ejection fraction, and myocardial mass, were calculated offline on a dedicated workstation (View Forum; Philips) from serial contiguous short-axis slices (usually 9-14). The same regions on the short-axis slices were defined as described for the PET study; myocardial thickness (ED and ES) and percentage systolic thickening were measured for each region $(16,31)$. Each myocardial segment was evaluated for the presence of DCE by 1 expert observer, unaware of the results of the other protocol investigations. DCE location, extent, and pattern (nontransmural or transmural) were analyzed (30). Volumes of DCE areas were calculated by planimetry in all short-axis slices, and the total volume of DCE was expressed as a percentage of total myocardium. Finally, the segments were grouped as follows: segments with DCE, either transmural (DCE-T) or nontransmural DCE (DCE-NT), and segments without DCE, either contiguous to DCE segments (NoDCE-C) or remote (NoDCE-R) from them.

\section{Statistical Analysis}

Continuous variables are shown as mean \pm SD. $\chi^{2}$ or Fisher exact tests, as appropriate, were used to compare noncontinuous variables expressed as proportions. The comparison of 2 datasets was performed by use of the Student $t$ test for unpaired data. In case of multiple comparisons, the 1-way ANOVA with the Scheffé post hoc test was used. The correlation between variables was evaluated using the Spearman $\rho$. All $P$ values are 2 -sided and considered significant when greater than 0.05 .

\section{RESULTS}

\section{PET and MRI Findings}

Mean hMBF for the entire left ventricle was $2.13 \pm 1.03$ $\mathrm{mL} / \mathrm{min} / \mathrm{g}$ (range, $0.97-5.57 \mathrm{~mL} / \mathrm{min} / \mathrm{g}$ ) and was comparable among patients in New York Heart Association (NYHA) functional class I versus those in class II/III $(1.9 \pm 0.9$ vs. $2.3 \pm 1.3 \mathrm{~mL} / \mathrm{min} / \mathrm{g}$, respectively; $P=0.23$ ). DCE was detected by MRI in 30 patients $(88 \%)$ and involved the segments with the greatest degree of hypertrophy (i.e., the interventricular septum) in all (Fig. 1); in 5 patients only, DCE extended to the free wall as well. Of a total of 578 segments, the number of those with DCE was 194 (34\%). The average total volume of DCE within the left ventricle was $21.0 \pm 22.2 \mathrm{~mL}$ (range, $3-80 \mathrm{~mL}$ ), and the percentage value of the total $\mathrm{LV}$ volume was $16.9 \% \pm 7.4 \%$ (range, $2 \%-30 \%)$. The extent of DCE was comparable among patients in NYHA functional class I versus those in class II/III $(20.0 \pm 24.1$ vs. $22.3 \pm 20.76 \mathrm{~mL}$, respectively; $P=0.81)$.

\section{Relationship Between hMBF and DCE}

Of the 194 DCE segments, 40 showed DCE-T and 154 showed DCE-NT. Of the remaining 384 segments without DCE, 204 were NoDCE-C and 180 were NoDCE-R. Average hMBF was significantly different between the group with and the group without DCE $(1.81 \pm 0.94$ vs. $2.13 \pm 1.11 \mathrm{~mL} / \mathrm{min} / \mathrm{g}$, respectively; $P<0.001$ ). Within DCE segments, hMBF was significantly lower in DCE-T than in DCE-NT segments $(1.43 \pm 0.52$ vs. $1.91 \pm 1.0 \mathrm{~mL} /$ $\mathrm{min} / \mathrm{g}$, respectively; $P<0.001$ ). Within NoDCE segments, hMBF in NoDCE-C was significantly lower than that in NoDCE-R segments $(1.98 \pm 1.10$ vs. $2.29 \pm 1.10 \mathrm{~mL} / \mathrm{min} / \mathrm{g}$, respectively; $P<0.01)$. Virtually identical average hMBF was registered between DCE-NT and NoDCE-C segments.

According to ANOVA, a significant overall difference in hMBF was observed among the 4 subgroups $(\mathrm{F}=8.6, P<$ 0.0001). In the post hoc comparisons, a significant difference was observed between NoDCE-R and all other subgroups and between NoDCE-C and DCE-T but was not observed between NoDCE-C and DCE-NT or between DCE-T and DCE-NT (Fig. 2). A significant inverse correlation between hMBF and percentage transmurality of DCE (Spearman $\rho=-0.17, P<0.0001$ ) was demonstrated. Finally, severe coronary microvascular dysfunction (average $\mathrm{hMBF}$ in the lowest tertile for the whole study group) was more prevalent among NoDCE-C than NoDCE-R segments ( $33 \%$ vs. $24 \%$, respectively; $P<0.05$ ).

\section{Impact on Regional Function}

Both ED and ES wall thickness values were significantly greater in segments with DCE than in those segments without DCE (Table 2). Conversely, percentage systolic wall thickening was significantly impaired in DCE segments (Table 2). A highly significant difference among the 4 subgroups was registered for all 3 parameters (ANOVA: $\mathrm{F}=56.5, P<0.00001 ; \mathrm{F}=35.5, P<0.00001 ;$ and $\mathrm{F}=$ 24.7, $P<0.00001$, for ED thickness, ES thickness, and systolic thickening, respectively). In the post hoc comparisons, systolic thickening was significantly reduced in the 2 subgroups of segments with DCE compared with those without DCE, but no internal differences within each group was demonstrated (i.e., NoDCE-R vs. NoDCE-C and DCE-T vs. DCE-NT segments) (Fig. 3). A significant direct correlation between $\mathrm{hMBF}$ and systolic thickening (Spearman $\rho=0.20, P<0.0001$ ) was evident. Conversely, the percentage transmurality of DCE showed a strong inverse relationship to systolic thickening (Spearman $\rho=-0.37$, $P<0.0001)$.

\section{DISCUSSION}

The present study investigated the relationship between coronary microvascular dysfunction and fibrosis in patients with HCM. DCE by MRI is presently considered the most accurate method to evaluate myocardial fibrosis in vivo. With its high spatial resolution, contrast MRI has the 

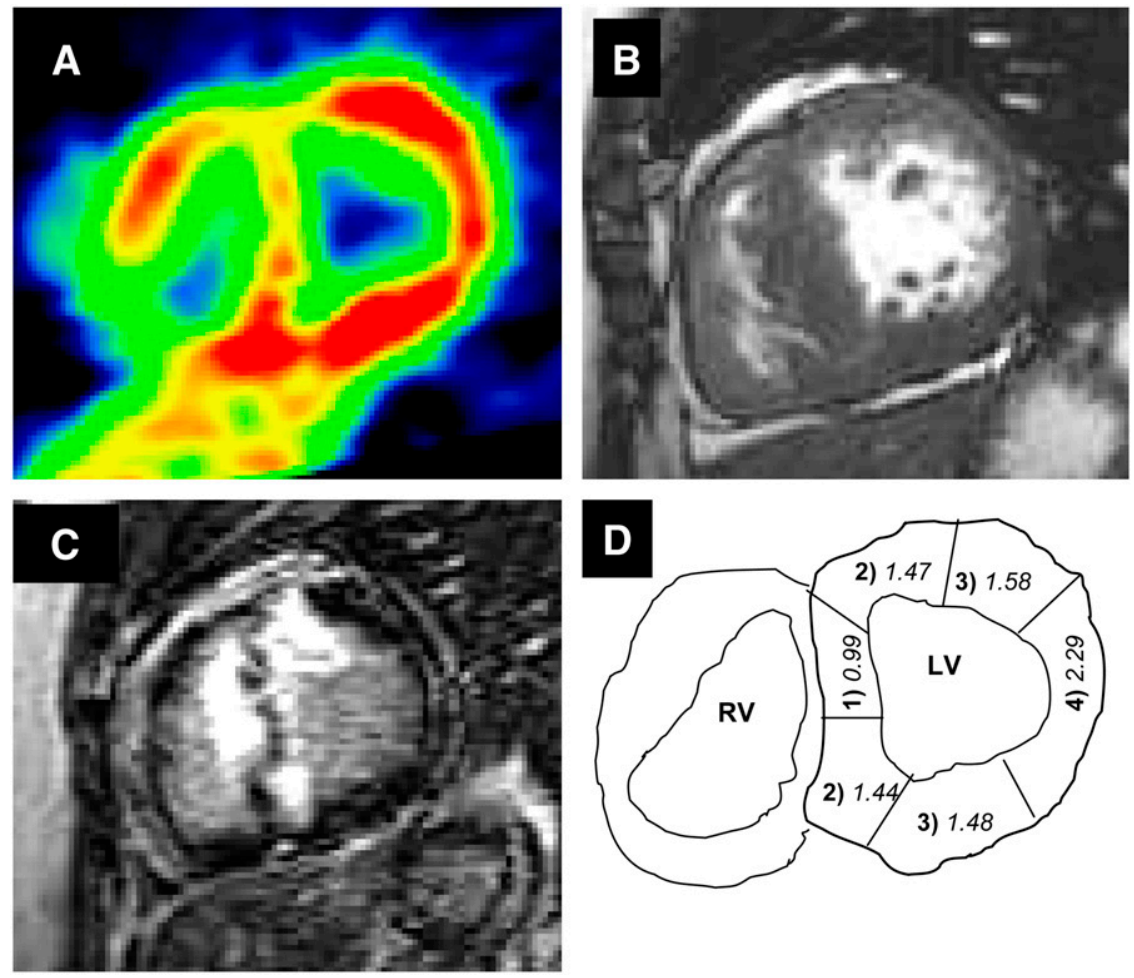

FIGURE 1. Relationship of hMBF to DCE in 28-y-old woman with HCM. (A) $\mathrm{NH}_{3}$ PET short-axis slice at level of basal LV segments. Color scale shows highest values of flow in red and lowest in green. (B) First-pass MRI short-axis slice at base of left ventricle, showing diffuse septal hypertrophy. (C) After gadolinium infusion, extensive DCE is evident (white signal), involving interventricular septum and extending into anterior wall. DCE and areas of reduced flow at PET closely agree. (D) Diagram illustrating nomenclature used for classification of myocardial segments with extent and proximity of DCE as follows: (1) transmural DCE, (2) nontransmural DCE, (3) without DCE but contiguous to DCE segments, (4) without DCE and remote from DCE. Numbers in italic indicate $\mathrm{hMBF}(\mathrm{mL} / \mathrm{min} / \mathrm{g})$ in segment. $\mathrm{RV}=$ right ventricle. unique advantage of allowing visualization of microinfarcts that cannot be detected by other noninvasive imaging techniques (32). In our patients, fibrosis was common and, in agreement with prior studies, had a marked preferential localization at the most hypertrophied segments and at midwall level $(15,16,33)$. This pattern clearly differs from that observed in patients with coronary artery disease because the latter is generally associated with reduced

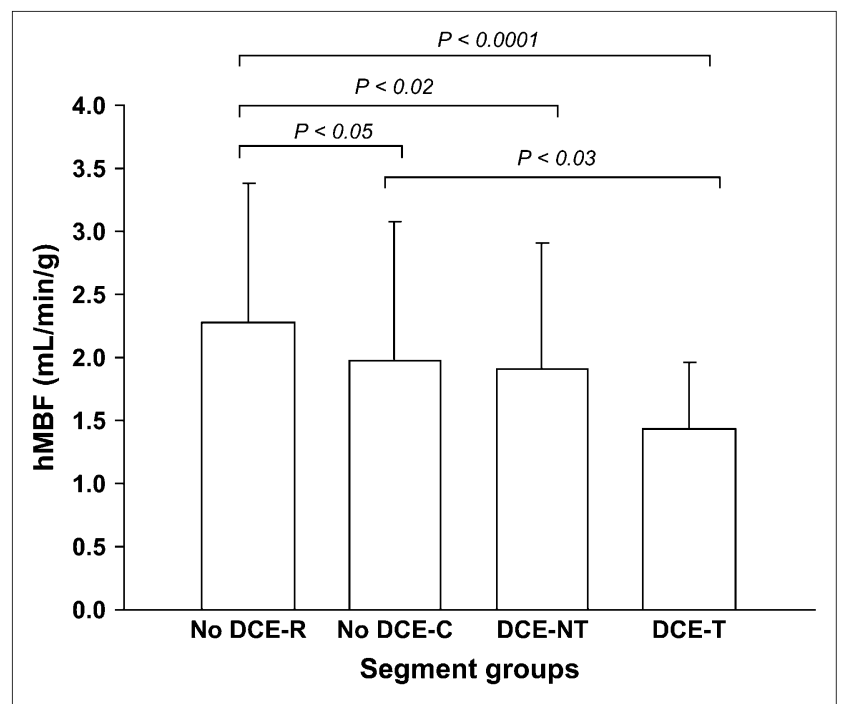

FIGURE 2. Bar graph of hMBF in LV segments grouped according to severity and proximity of DCE, showing statistical differences according to ANOVA post hoc test. myocardial wall thickness and always involves the subendocardial region $(32,34)$.

hMBF in segments without DCE is lower if they are located close to DCE segments than if the segments are remote from fibrotic areas. Average hMBF in these contiguous but DCE-free segments was virtually identical to that of segments with nontransmural DCE. These findings are consistent with the view that DCE may largely represent replacement fibrosis secondary to coronary microvascular dysfunction and ischemia. The additional observation of a strong relationship between impairment of hMBF, transmurality of DCE, and reduction in regional systolic wall thickening lends further corroboration to such a scenario.

Indeed, we demonstrated a continuum of coronary microvascular dysfunction ranging from regions with normal or near-normal wall thickness, in which hMBF is relatively preserved and DCE absent, to regions with greater hypertrophy, in which perfusion is increasingly impaired and DCE becomes progressively more frequent and extensive. Therefore, our data support the hypothesis that coronary microvascular dysfunction is a more widespread phenomenon than myocardial fibrosis in patients with HCM and may contribute to the development of fibrosis itself $(11,35$ 37).

Coronary microvascular dysfunction is a diffuse phenomenon in HCM and is not confined to the hypertrophied regions of the left ventricle $(12,18)$, although it does correlate in severity with the magnitude of segmental hypertrophy (33) and may have considerable regional heterogeneity $(13,18)$. Pathologic reports have demonstrated 
TABLE 2

Segmental ED and ES Wall Thickness and Systolic Thickening of the Segments With and Without DCE

\begin{tabular}{llrl}
\hline \multicolumn{1}{c}{ Parameter } & With DCE & Without DCE & $P$ \\
\hline ED wall thickness $(\mathrm{mm})$ & $16.4 \pm 7.8$ & $10 \pm 4.7$ & $<0.0001$ \\
ES wall thickness $(\mathrm{mm})$ & $21.7 \pm 8$ & $16.2 \pm 5.3$ & $<0.0001$ \\
Systolic thickening $(\%)$ & $42.5 \pm 38$ & $73 \pm 48$ & $<0.0001$ \\
\hline
\end{tabular}

that focal myocardial fibrosis, a common finding in patients with $\operatorname{HCM}(3,6,7)$, may be associated with evidence of ischemia (11). When marked, fibrosis is thought to represent one of the relevant substrates for end-stage progression and heart failure (35), as well as sudden cardiac death (36,37). Recently, Knaapen et al. observed that the extent of DCE in patients with HCM was inversely related to absolute resting perfusion, measured by ${ }^{15} \mathrm{O}$-labeled tracers (24). The authors concluded that regional heterogeneity of resting perfusion is related to the extent of fibrosis (24). In a previous study, however, the same authors had demonstrated that the water-perfusable tissue index was not decreased in the septum of patients with HCM with DCE and concluded that fibrosis is not the sole cause of DCE in these patients (23). Using MRI, Petersen et al. have shown that perfusion reserve is reduced in HCM independently of the presence of DCE (33). Our results expand the observation by Knaapen et al. (24) by demonstrating an inverse relationship between DCE severity and hMBF. Moreover, the finding that MBF may be reduced in segments without detectable DCE, significant hypertrophy, or systolic dysfunction but located close to DCE regions confirms that coronary microvascular dysfunction extends beyond the limits of DCE and probably precedes it. Therefore, our data and those by Petersen et al. (33) point to coronary microvascular dysfunction as a more diffuse abnormality than DCE in HCM and support the hypothesis that coronary microvascular dysfunction could contribute to the subse- quent development of fibrosis in HCM, probably mediated by recurrent ischemia. The opposite view that coronary microvascular dysfunction may be a consequence, rather than a cause, of myocardial fibrosis (24) may not be discounted in individual patients but appears to be less relevant in the general HCM population $(3-5,38)$. Further investigations are needed to demonstrate beyond doubt that coronary microvascular dysfunction is the main substrate for fibrosis in HCM. Moreover, different causes of DCE other than replacement fibrosis could be important in these patients and deserve additional studies (15).

Our results differ from those of Knaapen et al. about the interaction between MBF, DCE, and systolic function. Knaapen et al. demonstrated a significant inverse correlation between DCE extent and degree of regional systolic function and the lack of any relationship between resting $\mathrm{MBF}$ and regional function (24). In our series, we confirmed their findings by demonstrating an inverse relationship between DCE severity and ES thickening but also registered a significant direct correlation between this parameter and hMBF. Various explanations can be given for this apparent disagreement, including different methodologies in measuring microvascular and regional systolic function $(24,29,32)$. In particular, the use of systolic thickening in patients with hypertrophy can be problematic because the increased diastolic thickness can spuriously reduce this parameter. However, this parameter has already been used to assess regional function in the setting of HCM (16). Because patients with DCE have reduced wall thickening and thicker walls than do patients without DCE (39), hypertrophic segments with more DCE contract less effectively because of more extensive structural remodeling and not just because they are thicker.

Another technical limitation of our study is that we did not correct our hMBF measurements for the partial-volume effect. It must be considered, however, that the influence of the partial-volume effect on MBF measurements in patients with HCM is less critical than in healthy subjects, because

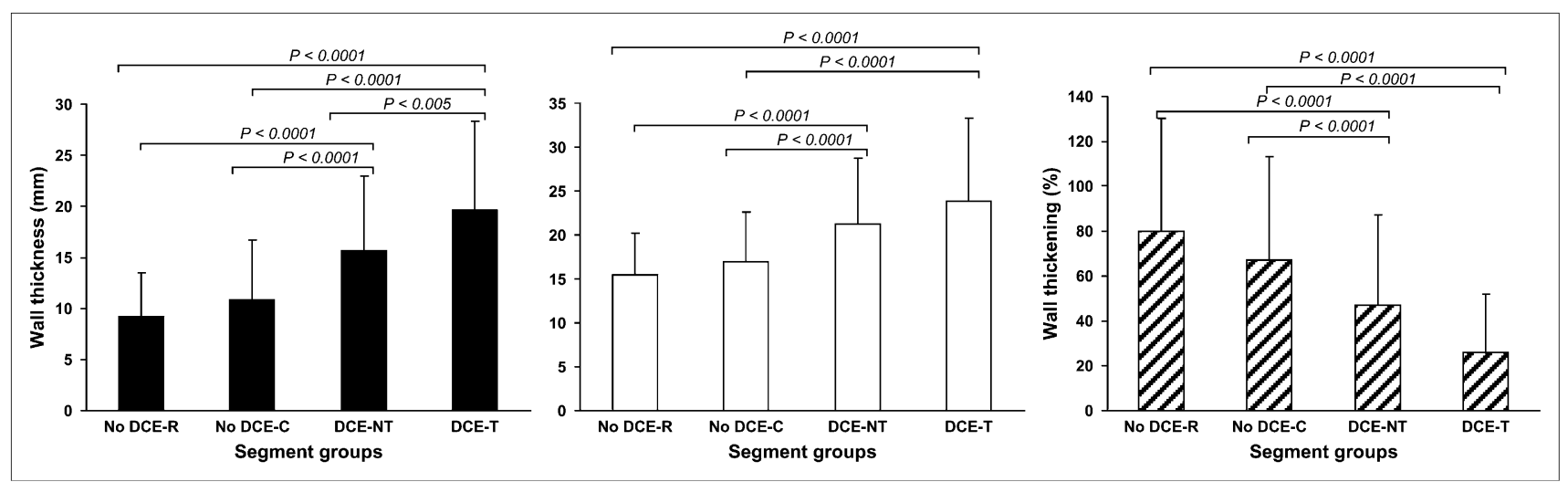

FIGURE 3. Bar graphs of ED wall thickness (black bars), ES wall thickness (white bars), and systolic thickening (hatched bars) in LV segments grouped according to severity and proximity of DCE, showing statistical differences according to ANOVA post hoc test. 
myocardial walls are thicker and frequently well above the PET scanner resolution (40). In our particular patient population with more extensive DCE in thicker segments, the choice not to perform the correction for the partialvolume effect can be regarded as conservative.

\section{CONCLUSION}

In our HCM cohort, hMBF was reduced in myocardial segments exhibiting DCE, especially when transmural. In segments without DCE, hMBF was blunted in those segments contiguous to DCE, showing values similar to those of nontransmural DCE segments. These results support the concept that increasing degrees of coronary microvascular dysfunction might play a causative role in the development of myocardial fibrosis in HCM.

\section{ACKNOWLEDGMENTS}

This study was supported by grants from the Italian Ministry for Scientific and Technologic Research (MURSTCOFIN 2004) and the Fondazione Ente Cassa di Risparmio di Firenze.

\section{REFERENCES}

1. Elliott P, McKenna W. Hypertrophic cardiomyopathy. Lancet. 2004;363:18811891.

2. Maron BJ. Hypertrophic cardiomyopathy: a systematic review. JAMA. 2002;287: 1308-1320.

3. Varnava AM, Elliott PM, Sharma S, McKenna WJ, Davies MJ. Hypertrophic cardiomyopathy: the interrelation of disarray, fibrosis, and small vessel disease. Heart. 2000;84:476-482.

4. Maron BJ, Wolfson JK, Epstein SE, Roberts WC. Intramural ("small vessel”) coronary artery disease in hypertrophic cardiomyopathy. J Am Coll Cardiol. 1986;8:545-557.

5. Tanaka M, Fujiwara H, Onodera T, et al. Quantitative analysis of narrowings of intramyocardial small arteries in normal hearts, hypertensive hearts, and hearts with hypertrophic cardiomyopathy. Circulation. 1987;75:1130-1139.

6. Factor SM, Butany J, Sole MJ, Wigle ED, Williams WC, Rojkind M. Pathological fibrosis and matrix connective tissue in the subaortic myocardium of patients with hypertrophic cardiomyopathy. J Am Coll Cardiol. 1991;17: 1343-1351.

7. Shirani J, Pick R, Roberts WC, Maron BJ. Morphology and significance of the left ventricular collagen network in young patients with hypertrophic cardiomyopathy and sudden cardiac death. J Am Coll Cardiol. 2000;35:36-44.

8. Maron BJ, Epstein SE, Roberts WC. Hypertrophic cardiomyopathy and transmural myocardial infarction without significant atherosclerosis of the extramural coronary arteries. Am J Cardiol. 1979;43:1086-1102.

9. Pasternac A, Noble J, Streulens Y, Elie R, Henschke C, Bourassa MG. Pathophysiology of chest pain in patients with cardiomyopathies and normal coronary arteries. Circulation. 1982;65:778-789.

10. Cannon RO III, Schenke WH, Maron BJ, et al. Differences in coronary flow and myocardial metabolism at rest and during pacing between patients with obstructive and patients with nonobstructive hypertrophic cardiomyopathy. $J \mathrm{Am}$ Coll Cardiol. 1987;10:53-62.

11. Basso C, Thiene G, Corrado D, Buja G, Melacini P, Nava A. Hypertrophic cardiomyopathy and sudden death in the young: pathologic evidence of myocardial ischemia. Hum Pathol. 2000;31:988-998.

12. Camici PG, Crea F. Coronary microvascular dysfunction. N Engl J Med. 2007; 356:830-840.

13. Cecchi F, Olivotto I, Gistri R, Lorenzoni R, Chiriatti G, Camici PG. Coronary microvascular dysfunction and prognosis in hypertrophic cardiomyopathy. N Engl J Med. 2003;349:1027-1035.

14. Olivotto I, Cecchi F, Gistri R, et al. Relevance of coronary microvascular flow impairment to long-term remodelling and systolic dysfunction in hypertrophic cardiomyopathy. J Am Coll Cardiol. 2006;47:1043-1048.
15. Moon JC, McKenna WJ, McCrohon JA, Elliott PM, Smith GC, Pennell DJ. Toward clinical risk assessment in hypertrophic cardiomyopathy with gadolinium cardiovascular magnetic resonance. J Am Coll Cardiol. 2003;41:1561-1567.

16. Choudhury L, Mahrholdt H, Wagner A, et al. Myocardial scarring in asymptomatic or mildly symptomatic patients with hypertrophic cardiomyopathy. $J \mathrm{Am}$ Coll Cardiol. 2002;40:2156-2164.

17. Kaufmann PA, Camici PG. Myocardial blood flow by PET: technical aspects and clinical applications. J Nucl Med. 2005;46:75-88.

18. Camici P, Chiaritti G, Lorenzoni R, et al. Coronary vasodilation is impaired in both hypertrophied and nonhypertrophied myocardium of patients with hypertrophic cardiomyopathy: a study with nitrogen-13 ammonia and positron emission tomography. J Am Coll Cardiol. 1991;17:879-886.

19. Camici PG, Cecchi F, Gistri R, et al. Dipyridamole-induced subendocardial underperfusion in hypertrophic cardiomyopathy assessed by positron emission tomography. Coron Artery Dis. 1991;2:837-841.

20. Choudhury L, Elliott P, Rimoldi O, et al. Transmural myocardial blood flow distribution in hypertrophic cardiomyopathy and effect of treatment. Basic Res Cardiol. 1999;94:49-59.

21. Lorenzoni R, Gistri R, Cecchi F, et al. Coronary vasodilator reserve is impaired in patients with hypertrophic cardiomyopathy and left ventricular dysfunction. Am Heart J. 1998;136:972-981.

22. Maron BJ, McKenna WJ, Danielson GK, et al. American College of Cardiology Foundation Task Force on Clinical Expert Consensus Documents, and European Society of Cardiology Committee for Practice Guidelines. American College of Cardiology/European Society of Cardiology Clinical Expert Consensus Document on Hypertrophic Cardiomyopathy. A report of the American College of Cardiology Foundation Task Force on Clinical Expert Consensus Documents and the European Society of Cardiology Committee for Practice Guidelines. Eur Heart J. 2003;24:1965-1991.

23. Knaapen P, van Dockum WG, Bondarenko O, et al. Delayed contrast enhancement and perfusable tissue index in hypertrophic cardiomyopathy: comparison between cardiac MRI and PET. J Nucl Med. 2005;46: 923-929.

24. Knaapen P, van Dockum WG, Götte MJ, et al. Regional heterogeneity of resting perfusion in hypertrophic cardiomyopathy is related to delayed contrast enhancement but not to systolic function: a PET and MRI study. J Nucl Cardiol. 2006;13:660-667.

25. Cheitlin MD, Alpert JS, Armstrong WF, et al. ACC/AHA guidelines for the clinical application of echocardiography. A report of the American College of Cardiology/American Heart Association Task Force on Practice Guidelines (Committee on Clinical Application of Echocardiography). Circulation. 1997; 95:1686-1744

26. Gistri R, Cecchi F, Choudhury L, et al. Effect of verapamil on absolute myocardial blood flow in hypertrophic cardiomyopathy. Am J Cardiol. 1994;74: 363-368.

27. Cardiac Modeling PMODE (PCARD). User guide. Available at: http://www. pmod.com/technologies/doc/pcard/toc.htm. Accessed May 30, 2008.

28. Cerqueira MD, Weissman NJ, Dilsizian V, et al. American Heart Association Writing Group on Myocardial Segmentation and Registration for Cardiac Imaging. Standardized myocardial segmentation and nomenclature for tomographic imaging of the heart: a statement for healthcare professionals from the Cardiac Imaging Committee of the Council on Clinical Cardiology of the American Heart Association. Circulation. 2002;105:539-542.

29. Hutchins GD, Schwaiger M, Rosenspire KC, Krivokapich J, Schelbert H, Kuhl DE. Noninvasive quantification of regional blood flow in the human heart using $\mathrm{N}-13$ ammonia and dynamic positron emission tomographic imaging. J Am Coll Cardiol. 1990;15:1032-1042.

30. Simonetti OP, Kim RJ, Fieno DS, et al. An improved MR imaging technique for the visualization of myocardial infarction. Radiology. 2001;218:215-223.

31. Ichikawa Y, Sakuma H, Suzawa N, et al. Late gadolinium-enhanced magnetic resonance imaging in acute and chronic myocardial infarction improved prediction of regional myocardial contraction in the chronic state by measuring thickness of nonenhanced myocardium. J Am Coll Cardiol. 2005;45:901-909.

32. Ricciardi MJ, Wu E, Davidson CJ, et al. Visualization of discrete microinfarction after percutaneous coronary intervention associated with mild creatine kinaseMB elevation. Circulation. 2001;103:2780-2783.

33. Petersen SE, Jerosch-Herold M, Hudsmith LE, et al. Evidence for microvascular dysfunction in hypertrophic cardiomyopathy: new insights from multiparametric magnetic resonance imaging. Circulation. 2007;115:24182425.

34. Wu E, Wu E, Judd RM, et al. Visualisation of presence, location, and transmural extent of healed Q-wave and non-Q-wave myocardial infarction. Lancet. 2001; 357:21-28. 
35. Harris KM, Spirito P, Maron MS, et al. Prevalence, clinical profile, and significance of left ventricular remodeling in the end-stage phase of hypertrophic cardiomyopathy. Circulation. 2006;114:216-225.

36. Varnava AM, Elliott PM, Mahon N, Davies MJ, McKenna WJ. Relation between myocyte disarray and outcome in hypertrophic cardiomyopathy. Am J Cardiol. 2001;88:275-279.

37. Varnava AM, Elliott PM, Baboonian C, Davison F, Davies MJ, McKenna WJ. Hypertrophic cardiomyopathy: histopathological features of sudden death in cardiac troponin T disease. Circulation. 2001;104:1380-1384.
38. Lombardi R, Betocchi S, Losi MA, et al. Myocardial collagen turnover in hypertrophic cardiomyopathy. Circulation. 2003;108:1455-1460.

39. Bogaert J, Goldstein M, Tannouri F, Golzarian J, Dymarkowski S. Original report: late myocardial enhancement in hypertrophic cardiomyopathy with contrast-enhanced MR imaging. AJR. 2003;180:981-985.

40. Yoshida K, Endo M, Himi T, et al. Measurement of regional myocardial blood flow in hypertrophic cardiomyopathy: application of the first-pass flow model using $\left[{ }^{13} \mathrm{~N}\right]$ ammonia and PET. Am J Physiol Imaging. 1989;4: 97-104.

\section{Erratum}

In the article "DPA-714, a New Translocator Protein-Specific Ligand: Synthesis, Radiofluorination, and Pharmacologic Characterization" by James ML, et al. (J Nucl Med. 2008;49:814-822), there was an error in the inclusion of Silvia Selleri as a coauthor. Barbara Costa (Department of Psychiatry, Neurobiology, Pharmacology, and Biotechnology, University of Pisa), as the actual person who conducted the in vitro screening, should have been listed as coauthor in place of Prof. Selleri. The authors regret the error. 